
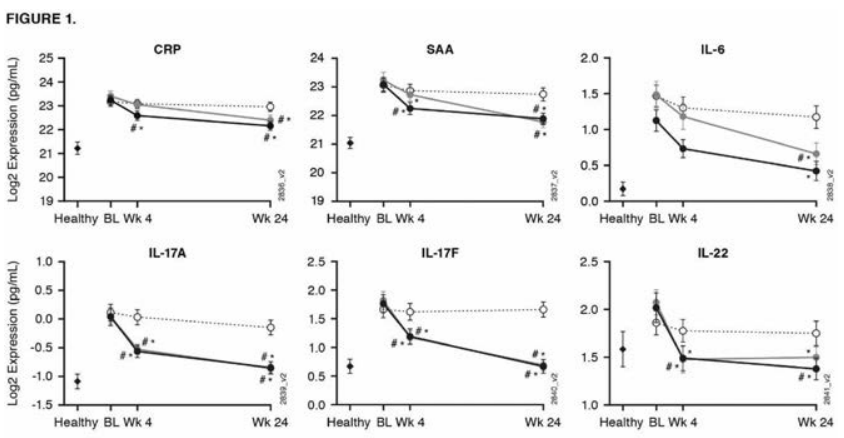

$\rightarrow$ GUS quw $\rightarrow$ GUS q8w O. PBO Healthy
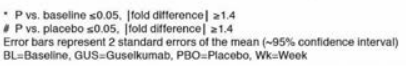

FIGURE 2.

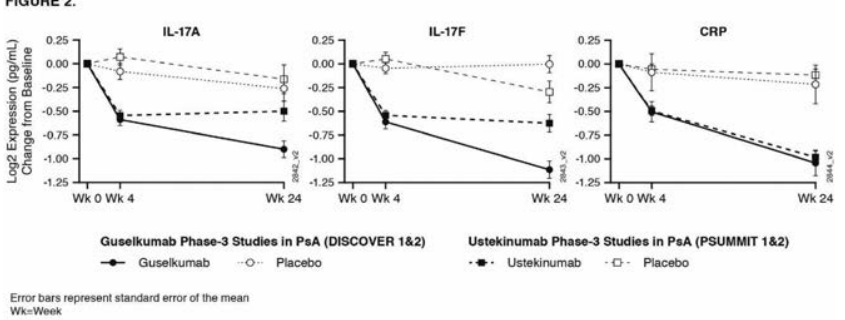

Acknowledgments: None

Disclosure of Interests: Stefan Siebert Grant/research support from: BMS, Boehringer Ingelheim, Celgene, GlaxoSmithKline, Janssen, Novartis, Pfizer, UCB, Consultant of: AbbVie, Boehringer Ingelheim, Janssen, Novartis, Pfizer, UCB, Speakers bureau: AbbVie, Celgene, Janssen, Novartis, lain Mclnnes Grant/ research support from: Bristol-Myers Squibb, Celgene, Eli Lilly and Company, Janssen, and UCB, Consultant of: AbbVie, Bristol-Myers Squibb, Celgene, Eli Lilly and Company, Gilead, Janssen, Novartis, Pfizer, and UCB, Matthew J Loza Employee of: Janssen Research \& Development, LLC, Keying Ma Employee of: Janssen Research \& Development, LLC, Karen Leander, Employee of: Janssen Research \& Development, LLC, Vani Lakshminarayanan Employee of: Janssen Research \& Development, LLC, Carol Franks Employee of: Janssen Research \& Development, LLC, Philip Cooper Employee of: Janssen Research \& Development, LLC, Kristen Sweet Employee of: Janssen Research \& Development, LLC DOI: 10.1136/annrheumdis-2020-eular.1240

\section{OP0230 EFFICACY AND SAFETY OF TILDRAKIZUMAB, A HIGH- AFFINITY ANTI-INTERLEUKIN-23P19 MONOCLONAL ANTIBODY, IN PATIENTS WITH ACTIVE PSORIATIC ARTHRITIS IN A RANDOMISED, DOUBLE-BLIND, PLACEBO-CONTROLLED, MULTIPLE-DOSE, PHASE 2B STUDY}

P. J. Mease ${ }^{1}$, S. Chohan ${ }^{2}$, F. J. García Fructuoso ${ }^{3}$, A. B. Gottlieb ${ }^{4}$, M. E. Luggen ${ }^{5}$, P. Rahman ${ }^{6}$, S. P. Raychaudhuri ${ }^{7}$, R. C. Chou $^{8}$, A. M. Mendelsohn ${ }^{9}$, S. Rozzo ${ }^{9}$, A. M. Orbai ${ }^{10}$. ${ }^{1}$ Swedish Medical Ctr/Providence St. Joseph Health and Univ of Washington, Seattle, United States of America; ${ }^{2}$ Arizona Arthritis and Rheumatology Research, PLLC, Phoenix, United States of America; ${ }^{3}$ Hospital CIMA Sanitas, Barcelona, Spain; ${ }^{4}$ Dept of Dermatology, Icahn School of Medicine at Mount Sinai, New York, United States of America; ${ }^{5}$ Cincinnati Rheumatic Disease Study Group, Inc, and Univ of Cincinnati College of Medicine, Cincinnati, United States of America; ${ }^{6}$ Craig L Dobbin Genetics Research Ctr, Memorial Univ, St. John's, Canada; ${ }^{7}$ Division of Rheumatology, Allergy \& Clinical Immunology, Univ of California School of Medicine, Davis, and VA Medical Ctr Sacramento, Sacramento, United States of America; ${ }^{8}$ Division of Allergy, Immunology and Rheumatology, Univ at Buffalo School of Medicine and Biomedical Sciences, Buffalo, United States of America; ${ }^{9}$ Sun Pharmaceutical Industries, Inc, Princeton, United States of America; ${ }^{10}$ Johns Hopkins Arthritis Ctr, Johns Hopkins Bayview Medical Ctr, Baltimore, United States of America

Background: Tildrakizumab (TIL), a high-affinity anti-interleukin-23p19 monoclonal antibody, is approved to treat moderate to severe plaque psoriasis and is under investigation for treatment of psoriatic arthritis (PsA). ${ }^{1}$

Objectives: To evaluate efficacy and safety of TIL up to week (W)52 in a randomised, double-blind, placebo-controlled, multiple-dose, phase $2 \mathrm{~b}$ study in PsA (NCT02980692).
Methods: Patients $(\mathrm{pts}) \geq 18$ years with active $\mathrm{PsA}^{2}$ were randomised 1:1:1:1:1 to TIL $200 \mathrm{mg}$ every 4 weeks (Q4W) to W52, TIL $200 \mathrm{mg}$ Q12W to W52, TIL $100 \mathrm{mg} \mathrm{Q12W}$ to W52, TIL $20 \mathrm{mg}$ Q12W until W24 then TIL $200 \mathrm{mg}$ Q12W to W52, or placebo (PBO) Q4W until W24 then TIL $200 \mathrm{mg}$ Q12W to W52. Efficacy assessments included ACR20/50/70, 75\%/90\%/100\% improvement in Psoriasis Area and Severity Index (PASI), proportion of pts with residual minimal disease activity (MDA) response; and mean change from baseline (BL) in HAQ-DI, Leeds Dactylitis Index (LDI, pts with BL LDI $\geq 1$ ), and Leeds Enthesitis Index (LEI, pts with $B L L E I \geq 1$ ) to W52. Treatment-emergent adverse events (TEAEs) were monitored.

Results: Of 500 pts screened, 391 were randomised and received $\geq 1$ dose of drug. Proportions of ACR20/50/70 responders were superior with TIL vs PBO through W24; after W24 rates of responses further increased for TIL $20 \rightarrow 200 \mathrm{mg}$ Q12W and PBO $\rightarrow 200 \mathrm{mg}$ Q12W through W52 (Figure 1, 2). Other efficacy results are shown in Table. Overall from $\mathrm{BL} \rightarrow \mathrm{W} 24 / \mathrm{W} 25 \rightarrow \mathrm{W} 52,50.4 \% / 39.9 \%$ and $2.3 \% / 1.0 \%$ of pts experienced a TEAE and serious $A E$, respectively. From $\mathrm{BL} \rightarrow \mathrm{W} 24,1$ case of pyelonephritis and urinary tract infection was reported in the TIL $100 \mathrm{mg}$ Q12W arm and 1 case of chronic tonsillitis was reported in the TIL 20 $\mathrm{mg} \rightarrow 200 \mathrm{mg} \mathrm{Q12W}$ arm. During W25 $\rightarrow$ W52, 1 malignancy (intraductal proliferative breast lesion) was reported with TIL $20 \mathrm{mg} \rightarrow 200 \mathrm{mg}$ Q12W. No deaths or major adverse cardiac events occurred.

Figure 1. ACR20/50/70 through W52
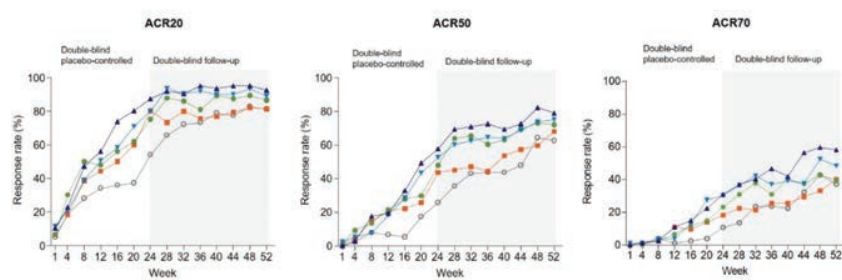

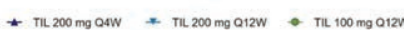

- TL 20,200 mg Q12W \& Placebo $\rightarrow$ TL. $200 \mathrm{mg}$ Q12W

Missing data were not imputed.
O4W, every 4 wooks: O12W, every 12 weeks: TIL, ilidrakizumat:

Figure 2. ACR20/50/70 through W24

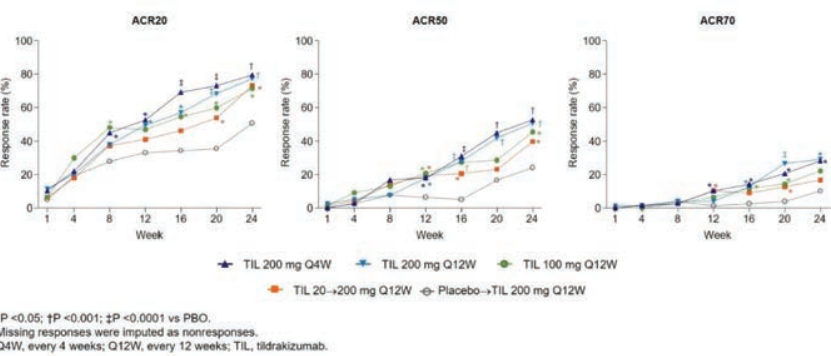

Table. W52 clinical efficacy

\begin{tabular}{|c|c|c|c|c|c|}
\hline & $\begin{array}{c}\text { TIL 200 mg } \\
\begin{array}{c}\text { Q4W } \\
n=78\end{array}\end{array}$ & $\begin{array}{c}\text { TIL 200 mg } \\
\text { Q12W } \\
n=79\end{array}$ & $\begin{array}{c}\text { TIL } 100 \mathrm{mg} \\
\text { Q12W } \\
n=77\end{array}$ & $\begin{array}{c}\text { TIL } 20 \rightarrow \\
200 \mathrm{mg} \mathrm{Q12W} \\
\mathrm{n}=78\end{array}$ & $\begin{array}{c}\mathrm{PBO} \rightarrow \\
\text { TIL 200 mg } \\
\text { Q12W } \\
n=79\end{array}$ \\
\hline HAQ-DI, BL ${ }^{a}$ & $1.0 \pm 0.6$ & $1.0 \pm 0.6$ & $1.0 \pm 0.7$ & $1.1 \pm 0.6$ & $1.2 \pm 0.6$ \\
\hline W5 $2^{\mathrm{b}}$ & $-0.5 \pm 0.5$ & $-0.5 \pm 0.6$ & $-0.5 \pm 0.6$ & $-0.5 \pm 0.5$ & $-0.5 \pm 0.5$ \\
\hline $\mathrm{LEI}, \mathrm{BL}^{\mathrm{a}, \mathrm{c}}$ & $1.9 \pm 2.0$ & $1.5 \pm 1.9$ & $2.2 \pm 2.1$ & $2.2 \pm 2.0$ & $1.5 \pm 1.9$ \\
\hline W52 $2^{\mathrm{b}}$ & $-1.3 \pm 1.9$ & $-1.0 \pm 1.6$ & $-1.7 \pm 2.1$ & $-1.2 \pm 1.8$ & $-1.2 \pm 1.8$ \\
\hline LDI, $\mathrm{BL}^{\mathrm{a}, \mathrm{d}}$ & $32.8 \pm 32.9$ & $61.3 \pm 73.5$ & $93.8 \pm 146.5$ & $71.4 \pm 118.5$ & $99.6 \pm 170.7$ \\
\hline W52 & $-21.4 \pm 37.1$ & $-42.1 \pm 76.7$ & $-41.6 \pm 89.3$ & $-56.5 \pm 123.4$ & $-81.5 \pm 173.0$ \\
\hline $\begin{array}{l}\text { BL, W52 } \\
\text { median }^{d}\end{array}$ & $21.8,7.4$ & 28.3, 3.2 & $32.1,20.0$ & 28.6, 0 & $34.0,5.6$ \\
\hline $\mathrm{MDA}^{\mathrm{e}}$ & 56.9 & 64.4 & 45.0 & 47.1 & 42.0 \\
\hline PASI $100^{\circ}$ & 54.0 & 44.4 & 43.9 & 47.5 & 35.0 \\
\hline PASI $90^{\circ}$ & 72.0 & 80.6 & 58.5 & 55.0 & 50.0 \\
\hline PASI $75^{\mathrm{e}}$ & 82.0 & 94.4 & 82.9 & 75.0 & 67.5 \\
\hline
\end{tabular}

${ }^{a} \mathrm{BL}$ mean $+\mathrm{SD} .{ }^{b}$ Mean change from $\mathrm{BL} \pm \mathrm{SD}$. ${ }^{\mathrm{c}} \mathrm{Pts}$ with $\mathrm{BL} \mathrm{LEI} \geq 1$ will be presented at EULAR. ${ }^{\mathrm{d}} \mathrm{Pts}$ with $\mathrm{BL} L \mathrm{LD} \geq 1(\mathrm{n}=27,21,21,19,25)$ using nonresponder imputation. ${ }^{{ }^{\circ}} \%$ at $\mathrm{W} 52$ Missing data not imputed.

$\mathrm{SD}$, standard deviation.

Conclusion: TIL was well tolerated and improved joint and skin manifestations of PsA through W52. 
References:

[1] Reich, et al. Lancet 2017;390:276-88.

[2] Taylor, et al. Arthritis Rheum 2006;54:2665-73.

Disclosure of Interests: Philip J Mease Grant/research support from: Abbott, Amgen, Biogen Idec, BMS, Celgene Corporation, Eli Lilly, Novartis, Pfizer, Sun Pharmaceutical, UCB - grant/research support, Consultant of: Abbott, Amgen, Biogen Idec, BMS, Celgene Corporation, Eli Lilly, Novartis, Pfizer, Sun Pharmaceutical, UCB - consultant, Speakers bureau: Abbott, Amgen, Biogen Idec BMS, Eli Lilly, Genentech, Janssen, Pfizer, UCB - speakers bureau, Saima Chohan Employee of: Partner/physician at Arizona Arthritis and Rheumatology Associates, Ferran J García Fructuoso Grant/research support from: AbbVie, Eli Lilly, Gedeon Richter, Medlmmune, Nichi-Iko, Pfizer, Sanofi-Aventis, Takeda, and UCB, Consultant of: AbbVie, Eli Lilly, Gedeon Richter, Medlmmune, NichiIko, Pfizer, Sanofi-Aventis, Takeda, and UCB, Speakers bureau: AbbVie, Eli Lilly, Gedeon Richter, Medlmmune, Nichi-Iko, Pfizer, Sanofi-Aventis, Takeda, and UCB, Alice B Gottlieb Grant/research support from:: Research grants, consultation fees, or speaker honoraria for lectures from: Pfizer, AbbVie, BMS, Lilly, MSD, Novartis, Roche, Sanofi, Sandoz, Nordic, Celltrion and UCB., Consultant of:: Research grants, consultation fees, or speaker honoraria for lectures from: Pfizer, AbbVie, BMS, Lilly, MSD, Novartis, Roche, Sanofi, Sandoz, Nordic, Celltrion and UCB., Speakers bureau:: Research grants, consultation fees, or speaker honoraria for lectures from: Pfizer, AbbVie, BMS, Lilly, MSD, Novartis, Roche, Sanofi, Sandoz, Nordic, Celltrion and UCB., Michael E Luggen Grant/ research support from: AbbVie; Amgen; Eli Lilly; Genentech; Nichi-Iko; Novartis; Pfizer; R-Pharm; and Sun Pharmaceutical Industries, Inc., Consultant of: AbbVie; Amgen; Eli Lilly; Genentech; Nichi-Iko; Novartis; Pfizer; R-Pharm; and Sun Pharmaceutical Industries, Inc., Speakers bureau: AbbVie; Amgen; Eli Lilly; Genentech; Nichi-Iko; Novartis; Pfizer; R-Pharm; and Sun Pharmaceutical Industries, Inc., Proton Rahman Grant/research support from: Janssen and Novartis, Consultant of: Abbott, AbbVie, Amgen, BMS, Celgene, Lilly, Janssen, Novartis, and Pfizer., Speakers bureau: Abbott, AbbVie, Amgen, BMS, Celgene, Lilly, Janssen, Novartis, Pfizer, Siba P Raychaudhuri Grant/research support from: AbbVie; Janssen; Novartis, Pfizer; Sun Pharmaceutical Industries, Inc, Consultant of: Amgen; Eli Lilly; Janssen; Novartis and Pfizer, Richard C Chou Consultant of: Sun Pharmaceutical Industries, Inc, Alan M Mendelsohn Shareholder of: Johnson and Johnson, Employee of: Sun Pharmaceutical Industries, Inc, Stephen Rozzo Employee of: Sun Pharmaceutical Industries, Inc, Ana-Maria Orbai Grant/research support from: Abbvie, Eli Lilly and Company, Celgene, Novartis, Janssen, Horizon, Consultant of: Eli Lilly; Janssen; Novartis; Pfizer; UCB. Ana-Maria Orbai was a private consultant or advisor for Sun Pharmaceutical Industries, Inc, not in her capacity as a Johns Hopkins faculty member and was not compensated for this service.

DOI: 10.1136/annrheumdis-2020-eular.3908

\section{Rheumatoid arthritis - non biologic treatment and small molecules}

\section{OP0231 COMPARATIVE EFFECTIVENESS OF JAK-INHIBITORS, TNF-INHIBITORS, ABATACEPT AND IL-6 INHIBITORS IN AN INTERNATIONAL COLLABORATION OF REGISTERS OF RHEUMATOID ARTHRITIS PATIENTS (THE “JAK-POT" STUDY)}

K. Lauper ${ }^{1}$, D. Mongin ${ }^{1}$, S. A. Bergstra ${ }^{2}$, D. Choquette ${ }^{3}$, C. Codreanu ${ }^{4}$,

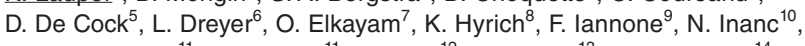
E. Kristianslund ${ }^{11}$, T. K. Kvien ${ }^{11}$, B. Leeb ${ }^{12}$, G. Lukina ${ }^{13}$, D. Nordström ${ }^{14}$, K. Pavelka ${ }^{15}$, M. Pombo-Suarez ${ }^{16}$, Z. Rotar ${ }^{17}$, M. J. Santos ${ }^{18}$, A. Strangfeld ${ }^{19}$, D. Courvoisier ${ }^{1}$, A. Finckh ${ }^{1} .{ }^{1} H U G$, Geneva, Switzerland; ${ }^{2}$ LUMC,

Rheumatology, Leiden, Netherlands; ${ }^{3}$ nstitut de Recherche en Rhumatologie, Montréal, Canada; ${ }^{4}$ Center of Rheumatic Diseases, Bucharest, Romania; ${ }^{5} \mathrm{KU}$ Leuven, Skeletal Biology and Engineering Research Unit, Leuven, Belgium; ${ }^{6}$ Aalborg University Hospital, DANBIO, Aalborg, Denmark; ${ }^{7}$ Tel Aviv University, Rheumatology, Tel Aviv, Israel; ${ }^{8}$ University of Manchester, Centre for Epidemiology Versus Arthritis, Manchester, United Kingdom; ${ }^{9}$ University Hospital of Bari, GISEA, Bari, Italy; ${ }^{10}$ Marmara University, Rheumatology, Istanbul, Turkey; ${ }^{11}$ Diakonhjemmet Hospital, Rheumatology, Oslo, Norway;

${ }^{12}$ State Hospital Stockerau, Rheumatology, Stockerau, Austria; ${ }^{13}$ V.A.Nasonova Research Institute of Rheumatology, Moscow, Russian Federation; ${ }^{14} \mathrm{Helsinki}$ University, ROB-FIN, Helsinki, Finland; ${ }^{15}$ Institute of Rheumatology, Prague, Czech Republic; ${ }^{16}$ Hospital Clinico Universitario, Rheumatology, Santiago de Compostela, Spain; ${ }^{17}$ biorx.si, Rheumatology, Ljubljana, Slovenia;

${ }^{18}$ Faculdade de Medicina, Reuma.pt, Lisboa, Portugal; ${ }^{19}$ DRFZ, Programme Area Epidemiology, Berlin, Germany
Background: In many countries, JAK-inhibitors (JAKi) have only recently been approved as treatment for patients with rheumatoid arthritis (RA).

Objectives: To evaluate the effectiveness of JAKi compared to bDMARDs in RA patients in the real-world population in an international collaboration of registers (the "JAK-pot" collaboration).

Methods: Patients initiating either JAKi, TNFi, IL-6i or abatacept (ABA) during a time period when JAKi were available in each country (19 registers, Table) were included. We compared the effectiveness of JAKi and bDMARDs in terms of retention using crude and adjusted survival analysis. Missing covariates were imputed using multiple imputation.

Results: Among 25521 included patients, 6063 initiated a JAKi, 13879 a TNFi, 2348 ABA, and 3231 an IL-6i. Patients were on average 55 years old, with a mean disease duration 10 years, mostly seropositive $(67 \%)$, female $(77 \%)$ and with moderate disease activity at treatment initiation. The main reason of stopping treatment was ineffectiveness (49\%), followed by adverse events (21\%). Patients on JAKi were treated more often as monotherapy, had higher CRP and disease activity at baseline and had experienced more previous ts/ bDMARDs. Crude median retention was 1.4 (95\% Cl 1.2-1.5) years for JAKi, 1.6 (1.6-1.7) for TNFi, 1.5 (1.3-1.7) for IL6i and 1.1 (1.0-1.3) for ABA. After adjustment, the hazard ratio $(\mathrm{HR})$ for discontinuation tended to be lower for JAKi (HR 0.86 (0.65-1.13)) compared to TNFi, but comparable for ABA (1.02 (0.94-1.10)) and IL6i $(0.99$ (0.88-1.10)) (Figure 1). HRs differed notably between countries (Figure 2).

Table 1. Registers

\begin{tabular}{lcc}
\hline Country, register & $\mathrm{N}$ & JAKi, n (\%) \\
\hline Austria, BIOREG* & & \\
Belgium, TARDIS & 6288 & $2113(33.6)$ \\
Canada, RHUMADATA & 528 & $114(21.6)$ \\
Czech Republic, ATTRA & 374 & $253(67.6)$ \\
Denmark, DANBIO & 4721 & $506(10.7)$ \\
Finland, ROB-FIN & 807 & $234(29.0)$ \\
Germany, RABBIT* & & \\
Italy, GISEA & 757 & $250(33.0)$ \\
Israel, I-RECORD & 400 & $94(23.5)$ \\
Netherlands, METEOR & 1642 & $4(0.2)$ \\
Norway, NOR-DMARD & 507 & $99(19.5)$ \\
Portugal, REUMA.PT & 797 & $44(5.5)$ \\
Romania, RRBR & 593 & $328(55.3)$ \\
Russia, ARBITER & 526 & $483(91.8)$ \\
Slovenia, BIORX.SI & 583 & $146(25.0)$ \\
Spain, BIOBADASER & 781 & $139(17.8)$ \\
Switzerland, SCQM & 2956 & $796(26.9)$ \\
Turkey, TURKBIO & 2150 & $397(18.5)$ \\
UK, BSRBR & 1111 & $63(5.7)$ \\
\hline
\end{tabular}

${ }^{*}$ Registers planning to participate in future studies but not included yet

Figure 1: Multivariable Cox model of drug discontinuation by type of treatment Ajusted for age, gender, disease duration, seropositivity, previous treatment, concomitant treatment, CRP, HAQ, CDA
and stratfified by country and year of treatment intiation. Strata - abatacept - TNFi - LL-6 inhibitors - JAK inhibitors

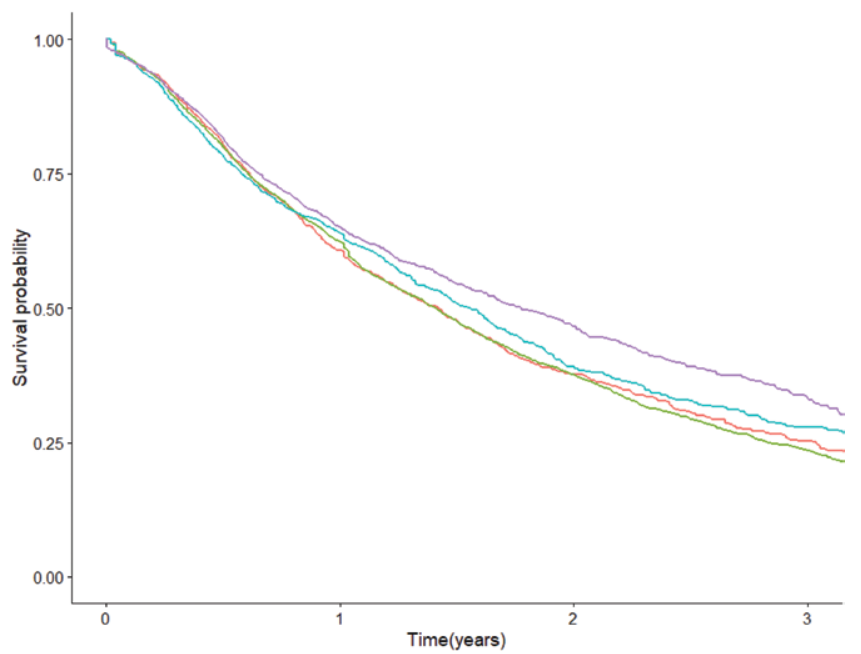

